\title{
Synthesis of some new tricyclic 1,4-benzothiazinones
}

\author{
M. B. Deshmukh ${ }^{*}$, S. S. Patil and A. R. Mulik \\ Department of Chemistry, Shivaji University, Kolhapur-416 004, India \\ E-mail : Mdeshmukh1@ rediffmail.com
}

Manuscript received 31 January 2001, revised 17 August 2001, accepted 16 October 2001

\begin{abstract}
2-Aminothiophenol on reaction with dimethyl oxalate and methyl 2-chloropropionate gives 1,4-benzothiazinone (1a, 1b) which when reacted with ethyl chloroacetate give $N$-ethoxycarbonylmethyl-1,4-benzothiazine-2,3-dione (2a $)$. Compound 2 on reaction with hydrazine hydrate affords $N$-hydrazidomethyl-1,4-benzothiazine-2,3-dione (3). Compound 3 is cyclized in pres-

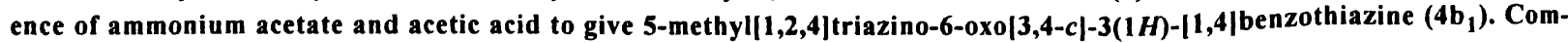
pound 1 on reaction with phosphorus oxychloride gives 3-chloro-1,4-benzothiazine-3-one (5a) which when reacts with ammonium sulfocyanide affords 1,4-benzothiazin-2-one-3-ylisocyanate (6a). Compound 6 when reacted with methyl cyanide gives the desired tricyclic compound 2-thio-6-methyl $|1,3,5|$ triazolo|3,4-c]benzothiazine-2-one (7a).
\end{abstract}

Benzothiazine derivatives exhibit various biological activities ${ }^{1}$. Here, we report the synthesis of some new tricyclic derivatives of benzothiazines. The strategy employed for the synthesis of desired heterocycles involved the $\mathrm{N}$-alkylation of benzothiazinone (1) with alkyl halo-esters to form $\mathrm{N}$ alkoxycarbonylalkylbenzothiazinones (2), which on reaction with hydrazine hydrate in methanol gave $\mathrm{N}$-hydrazidoalkylbenzothiazin-3(H)-ones (3). Compounds 3 were further cyclized in presence of ammonium acetate in acetic acid to give 1,2,4-triazino[3,4-c][1,4]benzothiazin-3(1H)-ones (4). Compound 1 in refluxing $\mathrm{POCl}_{3}$ gave 3-chloro-1,4benzothiazines (5) which when reacted with ammonium sulfocyanide gave 1,4-benzothiazin-2-one-3-yl-isocyanate (6). Compound 6 when subjected to Diels-Alder reaction with alkyl cyanide gave the targetted 2-thio-6-alkyl $[1,3,5]$ triazolo[3,4-c][1,4]benzothiazin-2-ones (7)(Scheme 1).

\section{Results and Discussion}

Benzothiazine-2,3-dione (1) was $N$-alkylated on reaction with ethylchloroacetate to form 2 . The disapperance of NH signal in 1 and appearance of a triplet and a quartet at $\delta$ 1.1 and 4.15 respectively due to $\mathrm{CH}_{3}$ and $\mathrm{OCH}_{2}$ confirmed the formation of ester $\mathbf{2}$. Compound $\mathbf{2}$ on reaction with hydrazine hydrate gave compound 3 , the formation of which was explained on the basis of the disappearance of ester $\mathrm{C}=\mathrm{O}$ encountered at $1735 \mathrm{~cm}^{-1}$ and appearance of amido $\mathrm{C}=\mathrm{O}$ at $1680 \mathrm{~cm}^{-1}$ in its IR spectrum. Compound 3 was cyclized in presence of ammonium acetate in acetic acid to get the tricyclic compound 4 . The formation of 4 was explained on the basis of the presence of $\mathrm{C}=\mathrm{N}$ band at 1610 $\mathrm{cm}^{-1}$ in its IR spectrum. Further, compound 1 was converted into its 3-chloro derivative (5) by refluxing in $\mathrm{POCl}_{3}$. The

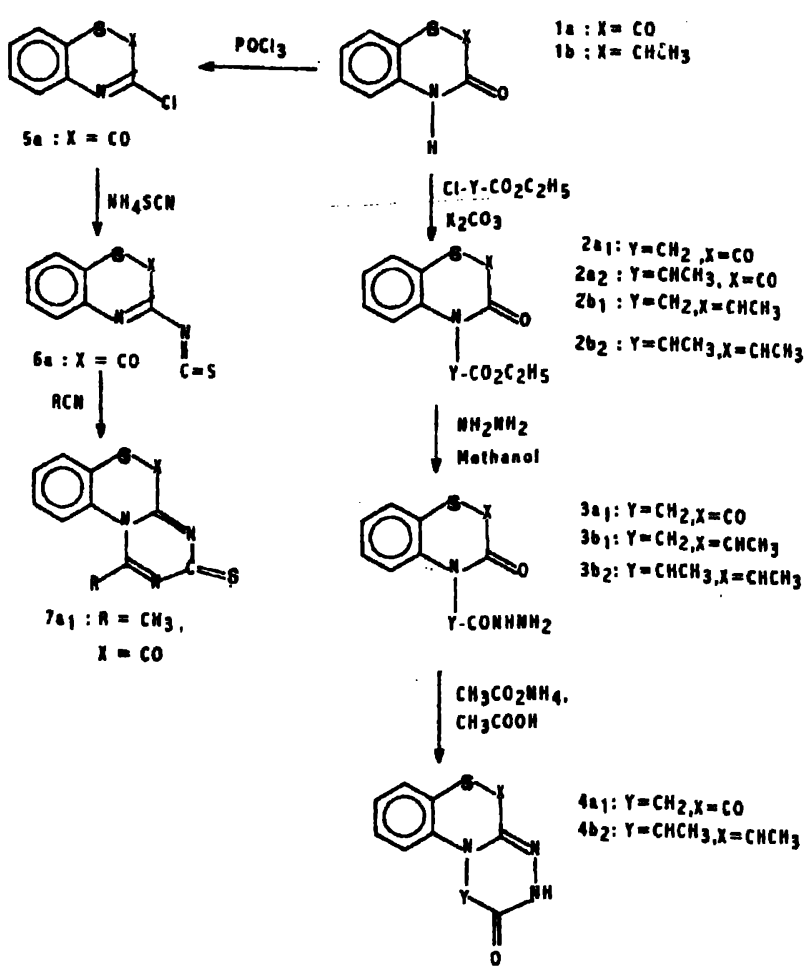

Scheme 1

formation of 5 was ensured by the disappearance of bands due to $\mathrm{N}-\mathrm{H}(3350)$ and $\mathrm{C}=\mathrm{O}\left(1680 \mathrm{~cm}^{-1}\right)$ and appearance of $\mathrm{C}=\mathrm{O}$ and $\mathrm{C}-\mathrm{Cl}$ bands at $1620(\mathrm{C}=\mathrm{O})$ and $760 \mathrm{~cm}^{-1}$ $(\mathrm{C}-\mathrm{Cl})$. Compound 5 on reaction with ammonium sulfocyanide yielded its isothiocyanate derivative 6 , which was confirmed by the appearance of IR band at $2050 \mathrm{~cm}^{-1}$ $(-\mathrm{N}=\mathrm{C}=\mathrm{S})$. Compound 6 was reacted with substituted alkyl cyanides to form the targetted tricyclic triazolo derivative 7 . The appearance of an additional signal at $\delta 2.188$ in its 
PMR spectrum and IR band at $1270-1190 \mathrm{~cm}^{-1}$ due to $\underset{\mathrm{N}}{\mathrm{CH}_{3}-\mathrm{C}}=\mathrm{N}$ and $\mathrm{C}=\mathrm{S}$.

\section{Experimental}

M.ps. were determined in open capillary tubes and are uncorrected. IR spectra $(\mathrm{KBr} /$ nujol) were recorded on a Perkin-Elmer 783 spectrophotometer and PMR spectra $\left(\mathrm{DMSO}-d_{6} / \mathrm{CDCl}_{3}\right.$ ) on a Brucker Ac $300 \mathrm{~F}$ spectrometer $(300 \mathrm{MHz})$ using TMS as an internal standard. Purity of the compounds was checked by TLC using silica gel G.

1,4-Benzothiazin-2,3-dione (1a) : A mixture of 2aminothiophenol $(2.915 \mathrm{~g}, 0.017 \mathrm{~mol})$ and dimethyl oxalate $(2.06 \mathrm{~g}, 0.017 \mathrm{~mol})$ in methanol $(20 \mathrm{ml})$ was refluxed for 5 $\mathrm{h}$ and then cooled. The separated solid was crystallized from DMF to get $1 \mathrm{a}(1.0 \mathrm{~g}, 32 \%)$, m.p. $260^{\circ} ; v_{\max } 3220-3140$ (NH), $1680(\mathrm{C}=\mathrm{O}), 1600 \mathrm{~cm}^{-1}(\mathrm{C}=\mathrm{C}) ; \delta 6.35-7.1(4 \mathrm{H}, \mathrm{m}$, $\mathrm{ArH}), 10.45(1 \mathrm{H}, \mathrm{s}, \mathrm{CONH}) .1 \mathrm{~b}$ was prepared by refluxing a mixture of 2-aminothiophenol ( $2 \mathrm{~g}, 0.016 \mathrm{~mol})$ and methyl 2-chloropropionate $(1.96 \mathrm{~g}, 0.016 \mathrm{~mol})$ in $33 \% \mathrm{NaOH}$ $(17.5 \mathrm{ml})$ on a water-bath for $5 \mathrm{~h}$. The reaction mixture was then cooled, neutralized with dil. $\mathrm{HCl}$, and the resulting solid was washed with water and crystallized from $80 \%$ ethanol, (70\%), m.p. 125\%; $v_{\max } 3220(\mathrm{NH}), 1700-1650 \mathrm{~cm}^{-1}$ (cyclic $\mathrm{C}=\mathrm{O}) ; \delta 1.5\left(3 \mathrm{H}, \mathrm{d}, \mathrm{CH}_{3}\right), 3.5-3.6(1 \mathrm{H}, \mathrm{q}, \mathrm{CH}), 6.95-$ 7.35 (4H, m, ArH), 9.2-9.3 (1H, s, NH).

$\mathrm{N}$-Ethoxycarbonylmethyl-1,4-benzothiazine-2,3-dione $\left(2 \mathrm{a}_{1}\right)$ : To a mixture of $1 \mathrm{a}(0.734 \mathrm{~g}, 0.0045 \mathrm{~mol})$ and ethyl chloroacetate $(1.12 \mathrm{~g}, 0.009 \mathrm{~mol})$ in acetone $(15 \mathrm{ml})$, potassium carbonate $(1 \mathrm{~g})$ was added. The reaction mixture was refluxed on an oil-bath for $36 \mathrm{~h}$, then cooled, filtered and the solvent removed under reduced pressure and the crude compound was column chromatographed using benzene as an eluent. The solvent was removed to get the solid $(1.0 \mathrm{~g}$, 89\%), m.p. 98 ; $v_{\max } 1740-1700$ (ester $\mathrm{C}=0$ ), 1700-1670 $\mathrm{cm}^{-1}$ (cyclic C=O); $\delta 1.1\left(3 \mathrm{H}, \mathrm{t}, J 7 \mathrm{~Hz}, \mathrm{CH}_{3}\right), 4.15(2 \mathrm{H}, \mathrm{q}$, $\left.J 7 \mathrm{~Hz}, \mathrm{OCH}_{2}\right), 5.2\left(2 \mathrm{H}, \mathrm{s}, \mathrm{N}-\mathrm{CH}_{2}\right), 7.15-7.5(4 \mathrm{H}, \mathrm{m}, \mathrm{ArH})$; $2 \mathrm{a}_{2}$ oil $(0.71 \mathrm{~g}, 40 \%) ; v_{\max } 1740$ (ester CO), 1700-1670 $\mathrm{cm}^{-1}$ (cyclic amido $\mathrm{CO}$ ); $\delta 1.1\left(3 \mathrm{H}, \mathrm{t}, J 7 \mathrm{~Hz}, \mathrm{CH}_{3}\right), 1.5$ $\left(3 \mathrm{H}, \mathrm{d}, J 7 \mathrm{~Hz}, \mathrm{CHCH}_{3}\right), 3.2(1 \mathrm{H}, \mathrm{q}, J 7.5 \mathrm{~Hz}, \mathrm{CH}), 4.18$ $\left(2 \mathrm{H}, \mathrm{q}, J 7 \mathrm{~Hz}, \mathrm{OCH}_{2}\right), 5.15\left(2 \mathrm{H}, \mathrm{s}, \mathrm{NCH}_{2}\right), 7.15-7.5(4 \mathrm{H}$, $\mathrm{m}, \mathrm{ArH}) ; 2 \mathrm{~b}_{1}$ oil ( $1.0 \mathrm{~g}, 84 \%$ ); $v_{\max } 1740-1735$ (ester $\mathrm{CO}$ ), $1680 \mathrm{~cm}^{-1}$ (cyclic amido CO); $\delta 1.5(3 \mathrm{H}, \mathrm{d}, J 7 \mathrm{~Hz}, \mathrm{CH}-$ $\left.\mathrm{CH}_{3}\right), 3.2(1 \mathrm{H}, \mathrm{q}, J 7 \mathrm{~Hz}, \mathrm{CH}), 4.15(2 \mathrm{H}, \mathrm{q}, J 7.5 \mathrm{~Hz}$, $\left.\mathrm{OCH}_{2}\right), 5.15\left(2 \mathrm{H}, \mathrm{s}, \mathrm{NCH}_{2}\right), 7.1-7.5(4 \mathrm{H}, \mathrm{m}, \mathrm{ArH}) ; 2 \mathrm{~b}_{2}$ oil $(0.9 \mathrm{~g}, 84 \%) ; v_{\max } 1740-1730$ (ester CO), $1680 \mathrm{~cm}^{-1}$ (cyclic amido $\mathrm{CO}) ; \delta 1.1\left(3 \mathrm{H}, \mathrm{t}, J 7 \mathrm{~Hz}, \mathrm{CH}_{3}\right), 1.5(3 \mathrm{H}, \mathrm{d}$, $\left.J 7 \mathrm{~Hz}, \mathrm{CH}_{3}\right), 4.18\left(2 \mathrm{H}, \mathrm{q}, J 7.5 \mathrm{~Hz}, \mathrm{OCH}_{2}\right), 5.1(1 \mathrm{H}, \mathrm{q}, J$
$7 \mathrm{~Hz}, \mathrm{NCH}), 7.15-7.55$ (4H, m, ArH).

$\mathrm{N}$-Hydrazidomethyl-1,4-benzothiazin-2,3-dione $\left(3 \mathrm{a}_{1}\right)$ : A mixture of $2 \mathrm{a}_{1}(0.1 \mathrm{~g}, 0.00037 \mathrm{~mol})$ and hydrazine hydrate $(0.02 \mathrm{~g}, 0.00037 \mathrm{~mol})$ in methanol $(10 \mathrm{ml})$ was refluxed on a steam-bath for $3 \mathrm{~h}$, then concentrated, cooled and filtered to obtain the product $(0.08 \mathrm{~g}, 85 \%)$, m.p. $125^{\circ}$; $v_{\max } 3300(\mathrm{NH}), 1700-1630$ (cyclic and acyclic $\mathrm{C}=\mathrm{O}$ ), 1600 $\mathrm{cm}^{-1}(\mathrm{C}=\mathrm{C}) ; \delta\left(\mathrm{DMSO}_{-} d_{6}\right) 4.6\left(2 \mathrm{H}, \mathrm{s}, \mathrm{NH}_{2}\right), 5.1(2 \mathrm{H}, \mathrm{s}$, $\left.\mathrm{N}-\mathrm{CH}_{2}\right), 7.15-7.5(4 \mathrm{H}, \mathrm{m}, \mathrm{ArH}), 10.45(1 \mathrm{H}, \mathrm{s}$, exchangable with $\left.\mathrm{D}_{2} \mathrm{O}, \mathrm{CONH}\right) ; 3 \mathrm{~b}_{1}(85 \%)$, m.p. $125^{\circ}, v_{\max } 3350-3150$ $\left(\mathrm{NHNH}_{2}\right.$ ), 1685 (acyclic amido $\mathrm{CO}$ ), $1670-1655 \mathrm{~cm}^{-1}$ (cyclic CO); $\delta 1.5\left(3 \mathrm{H}, \mathrm{d}, J 7 \mathrm{~Hz}, \mathrm{CH}_{3}\right), 3.2(1 \mathrm{H}, \mathrm{q}, J 7 \mathrm{~Hz}$, $\mathrm{CH}), 4.2\left(2 \mathrm{H}, \mathrm{s}\right.$ br, $\mathrm{NH}_{2}$ exchangable with $\left.\mathrm{D}_{2} \mathrm{O}\right), 7.1-7.55$ $(4 \mathrm{H}, \mathrm{m}, \mathrm{ArH}), 11.5(1 \mathrm{H}, \mathrm{s}$ br, $\mathrm{CONH}$, exchangable with $\left.\mathrm{D}_{2} \mathrm{O}\right) ; 3 \mathrm{~b}_{2}(62 \%)$, m.p. $127^{\circ} ; v_{\max } 3400\left(\mathrm{NHNH}_{2}\right), 1690$ 1670 (cyclic and acyclic $\mathrm{C}=\mathrm{O}$ ), $1600 \mathrm{~cm}^{-1}(\mathrm{C}=\mathrm{C}) ; \delta 1.12$ $\left(3 \mathrm{H}, \mathrm{t}, J 7 \mathrm{~Hz}, \mathrm{CH}_{3}\right), 1.5\left(3 \mathrm{H}, \mathrm{d}, J 7 \mathrm{~Hz}, \mathrm{CH}_{3}\right), 3.3(1 \mathrm{H}, \mathrm{q}$, $J 7 \mathrm{~Hz}, \mathrm{CH}), 4.18\left(2 \mathrm{H}, \mathrm{s}\right.$ br, $\mathrm{NH}_{2}$ exchangable with $\left.\mathrm{D}_{2} \mathrm{O}\right)$, $5.1(1 \mathrm{H}, \mathrm{q}, J 7 \mathrm{~Hz}, \mathrm{~N}-\mathrm{CH}), 7.15-7.5(4 \mathrm{H}, \mathrm{m}, \mathrm{ArH}), 11.5$ $\left(1 \mathrm{H}, \mathrm{s}\right.$ br, CONH exchangable with $\left.\mathrm{D}_{2} \mathrm{O}\right)$.

5-Methyl-[1,2,4]-triazino-6-oxo[3,4-c]-3(1H)-[1,4]benzothiazine $\left(\mathbf{4 b}_{1}\right)$ : A mixture of $3 \mathrm{~b}_{1}(0.06 \mathrm{~g}, 0.00024$ $\mathrm{mol})$ and ammonium acetate $(0.11 \mathrm{~g}, 0.0014 \mathrm{~mol})$ in acetic acid $(5 \mathrm{ml})$ was refluxed in an oil-bath till yellow colour of reaction mixture changed to dark-brown. Then the reaction mixture was poured on crushed-ice and neutralized with ammonia. The separated solid was washed with water and crystallized from ethanol, $(0.05 \mathrm{~g}, 87 \%)$, m.p. $145^{\circ}, v_{\max }$ 3400-3200 (NH), 1740-1630 (cyclic and acyclic $\mathrm{C}=\mathrm{O}$ ), $1600 \mathrm{~cm}^{-1}(\mathrm{C}=\mathrm{N}) ; \delta 1.1\left(3 \mathrm{H}, \mathrm{d}, J 7 \mathrm{~Hz}, \mathrm{CH}_{3}\right), 3.2(1 \mathrm{H}, \mathrm{q}$, $J 7 \mathrm{~Hz}, \mathrm{CH}), 5.18\left(2 \mathrm{H}, \mathrm{s}, \mathrm{NCH}_{2}\right), 7.15-7.55(4 \mathrm{H}, \mathrm{m}, \mathrm{ArH})$, $11.4\left(1 \mathrm{H}, \mathrm{s}\right.$ br, CONH exchangable with $\left.\mathrm{D}_{2} \mathrm{O}\right) ; 4 \mathrm{a}_{1}(78 \%)$; $v_{\text {max }}$ 3180-3100 (NH), 1690-1640 (cyclic C=O), 1595 $\mathrm{cm}^{-1}(\mathrm{C}=\mathrm{O}) ; \delta 5.1\left(2 \mathrm{H}, \mathrm{s}, \mathrm{N}-\mathrm{CH}_{2}\right), 7-0-7.55(4 \mathrm{H}, \mathrm{m}, \mathrm{ArH})$, $10.5\left(1 \mathrm{H}, \mathrm{s}\right.$, exchangable with $\left.\mathrm{D}_{2} \mathrm{O}, \mathrm{CONH}\right)$.

3-Chloro-1,4-benzothiazine-2-one (5a): A mixture of $1 \mathrm{a}(0.268 \mathrm{~g}, 0.0015 \mathrm{~mol})$ and phosphorus oxychloride $(0.0044 \mathrm{~mol})$ was refluxed on an oil-bath at $130-140^{\circ}$ for 5 $h$, then cooled, concentrated and poured on crushed-ice. The separated solid was washed with water and crystallised from benzene, $(0.2 \mathrm{~g}, 68 \%)$, m.p. $275^{\circ} ; v_{\max } 1710$ (lactone $\mathrm{C}=\mathrm{O}$ and amido $\mathrm{C}=\mathrm{O}), 1620(\mathrm{C}=\mathrm{N}), 760 \mathrm{~cm}^{-1}(\mathrm{C}-\mathrm{Cl}) ; \delta 7.1-7.5$ (4H, m, ArH).

1,4-Benzothiazin-2-one-3-yl isocyanate (6a) : A mixture of $5 \mathrm{a}(0.035 \mathrm{~g}, 0.003 \mathrm{~mol})$ ammonium sulfocyanide $(0.228 \mathrm{~g}, 0.003 \mathrm{~mol})$ in acetone $(5 \mathrm{ml})$ was stirred under reflux for $1.5 \mathrm{~h}$, then cooled, filtered and concentrated under vaccum. The residue was washed with water and 
crystallised from ethanol, (46\%), m.p. $190^{\circ} ; v_{\max } 1705$ $(\mathrm{C}=\mathrm{O}), 1620\left(\mathrm{C}^{\circ}=\mathrm{N}\right), 1600(\mathrm{C}=\mathrm{C}), 2050 \mathrm{~cm}^{-1}(-\mathrm{N}=\mathrm{C}=\mathrm{S}) ; \delta$ 7.1-7.55 (4H, m, ArH).

2-Thio-6-methyl $[1,3,5]$ triazolo $[3,4-\mathrm{c}][1,4]$ benzothiazin-2-one (7a) : To a solution of $6 \mathrm{a}(0.06 \mathrm{~g}, 0.0003$ $\mathrm{mol})$ in ethanol $(5 \mathrm{ml})$, methyl cyanide $(0.02 \mathrm{~g}, 0.0005 \mathrm{~mol})$ was added and the mixture refluxed on a steam-bath for $5 \mathrm{~h}$, then cooled, the solvent removed under reduced pressure and the residual oily mass triturated with ice-water to get reddish-brown product $(0.05 \mathrm{~g}, 64 \%)$, m.p. $139^{\circ} ; v_{\max } 1730$ $(\mathrm{C}=0), 1270-1190(\mathrm{C}=\mathrm{S}), 1600 \mathrm{~cm}^{-1}(\mathrm{C}=\mathrm{N}) ; \delta(\mathrm{DMSO}-$ $\left.d_{6}\right) 2.18\left(3 \mathrm{H}, \mathrm{s},=\mathrm{C}-\mathrm{CH}_{3}\right), 7.0-7.6(4 \mathrm{H}, \mathrm{m}, \mathrm{ArH})$.

\section{Acknowledgement}

The authors thank R.S.I.C., Punjab University, Chandigarh for PMR and IR analyses.

\section{References}

1 M. B. Hogale and A. C. Uthale, Indian J. Chem., Sect. B, 1990, 29, 590; V. L. Cizej and U. Urleb, $J$ Heterocycl. Chem., 1996, 33, 97 and the references cited thereın; M. B. Deshmukh (Hogale), J. S. Jadhav and S. H. Patil, J. Indian Chem. Soc., 1997, 74, 52; S H. Mashraqui, M. M. Biswas and K. R. Nivalkar, Indian J. Chem., Sect. B, 1996, 35, 1031; A. Napolitano, S Memoli and G. Prota, J. Org. Chem., 1999, 64, 3009; V. K. Ahluwalia, R. Aggarwal and R. Kumar, Indian J. Chem., Sect. B, 1994, 33, 65; B. R. Chaudhari, D. B. Shinde and M. S. Shingare, Indian J. Heterocycl. Chem., 1995, 4, 187. 